\title{
THE FIRST AND SECOND VARIATIONS OF AN $n$-TUPLE INTEGRAL IN THE CASE OF VARIABLE LIMITS*
}

\author{
BX \\ H. A. SIMMONS
}

1. Introduction. The main purpose of this paper is to generalize except in one detail all of the results which we obtained relative to a double integral in a previous article. $\dagger$ The first eight sections here would constitute a complete generalization of those results if the integrand of the $(n-1)$-tuple integral in equation (6.9) below could be expressed as a linear homogeneous function of $\zeta$ and not involve the $\zeta_{x_{i}}$ (cf. equation (49) of the previous article). We have not been able to do this. A special device that was used in obtaining equation (49) of the previous article does not seem capable of generalization here. In $\$ 9$ we suggest for the fundamental formulas in (4.8), for the integrals $J^{\prime}(0)$ and $J^{\prime \prime}(0)$, certain applications that are not made in $\$ \S 1-8$.

In view of certain papers of Lichtenstein $\ddagger$ and Reid§, in which Jacobi's condition is stated in terms of characteristic numbers of boundary value problems somewhat like the problem of $\$ 7$ below, and also because of recent advances in the theory of elliptic partial differential equations || our generalization seems desirable.

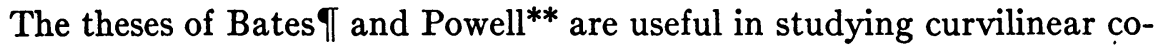
ordinate systems of the type that we employ here. We take the lines of curvature as the parameter lines.

The legitimacy of the use that we make of an extended Green's theorem is well known. $\dagger \dagger$

In this paper the variables $x_{1}, \cdots, x_{n}$ are the coordinates of a euclidean space $X$ of $n(n \geqq 2)$ dimensions in the euclidean space $X Z$ of $(n+1)$ dimensions of coordinates $x_{1}, \cdots, x_{n}, z$. An equation $z=z(x)$ or $\phi(x, z)=0$, where

* Presented to the Society, December 1, 1933; received by the editors March 29, 1933.

$\dagger$ These Transactions, April, 1926, p. 235.

$\ddagger$ Monatshefte für Mathematik und Physik, vol. 28 (1917), p. 3; Mathematische Zeitschrift, vol. 5 (1919), p. 26.

\& American Journal of Mathematics, vol. 54 (1932), p. 791.

\| Cf. bibliography at the close of Raab's thesis, Jacobi's condition ..., The University of Chicago Press.

I These Transactions, vol. 12 (1911), p. 19.

** The University of Chicago Press.

t† Cf., for example, Franklin, Annals of Mathematics, 1923, p. 213. 
$x \equiv\left(x_{1}, \cdots, x_{n}\right)$, defines an $n$-dimensional hypersurface in $X Z$. We let $p_{i} \equiv \partial z / \partial x_{i}(i=1, \cdots, n)$ and let $W$ stand for a $(2 n+1)$-dimensional open region in the space $X Z P$ of the variables $x, z$, and $p \equiv\left(p_{1}, \cdots, p_{n}\right)$. Then we define an admissible hypersurface $z=z(x)$ to be one with its elements in $W$ and having the following four properties: (i) $z$ is a single-valued function of the $x$ 's; (ii) $z$ is of class $C^{\prime \prime}$; (iii) it has a real, simply closed $(n-1)$-dimensional intersection (that is, a connected $(n-1)$-dimensional intersection that is bounded, closed, and does not intersect itself) $L_{0}^{\prime}$, with a fixed hypersurface $\phi(x, z)=0$, which is of class $C^{\prime \prime}$ and has no singular point for $x$ and $z$ in $W$; (iv) it is such that the projection, $L_{0}$, of $L_{0}^{\prime}$ on $X$ is met by a line parallel to any one of the coordinate axes, $x_{i}$, in a finite number of points and segments.

Property (iii) indicates the sense in which we use the term variable limits. The manifold $L_{0}^{\prime}$ is the boundary of the portion of the admissible hypersurface $z=z(x)$ that we consider. On account of property (i), the correspondence between points of $L_{0}$ and $L_{0}^{\prime}$ is one-to-one; and so $L_{0}$ is also a simply closed $(n-1)$-dimensional manifold (cf. (iii)). It bounds a simply connected portion of $X$ space. This we call $S_{0}$.

Property (iv) is required to insure that our application to $L_{0}$ and $S_{0}$ of an extended Green's theorem in $\S \S 5,7$ below shall be legitimate.

We consider here the $n$-tuple integral $(n \geqq 2)$

$$
I=\int_{S_{0}}^{n} f(x, z, p) d x,
$$

where $x, z, p, S_{0}$ are as defined above, and $f$ is of class $C^{\prime \prime \prime}$ in $W$. This integral $I$ is our generalization of the double integral

$$
\iint_{A_{0}} f(x, y, z, p, q) d x d y
$$

of the previous article.

Assuming that $z=z(x)$ is a minimizing admissible hypersurface for the integral $I$, we let $\zeta(x)$ be any function of the $x$ 's with properties (i), (ii), and such that if $a$ is a real parameter sufficiently small numerically, then $z=z(x)$ $+a \zeta(x)$ is admissible. Let $L_{a}^{\prime}$ denote the $(n-1)$-dimensional manifold

$$
\phi(x, z(x)+a \zeta(x))=0, \quad z=z(x)+a \zeta(x)
$$

when $x$ is sufficiently near the $x$ 's that determine points of $S_{0}{ }^{*}$; let $L_{a}$ denote the projection on $X$ space of $L_{a}^{\prime}$; and designate by $S_{a}$ the hyperarea in the $X$ space that is bounded by $L_{a}$. Then in place of the integral (1.1), we have $I(a)$ :

\footnotetext{
* So near that the correspondence between points $x$ and points $u v$ in $\S 2$ is $(1,1)$, reversible.
} 


$$
I(a)=\int_{S_{a}}^{n} f\left(x, z+a \zeta, p+a \zeta_{x}\right) d x .
$$

Our main problem is to obtain the first and second derivatives $I^{\prime}(0)$ and $I^{\prime \prime}(0)$, which are analogous to corresponding integrals of the previous article. We assume that $f \neq 0$ on the hypersurface $z=z(x)$ along its intersection $L_{0}^{\prime}$ with the fixed hypersurface $\phi(x, z)=0$ for a reason analogous to that which made a similar assumption desirable in the previous article.

In $\$ 2$, we set up a normal curvilinear coordinate system which plays an important rôle in later sections of this paper; in \$3, the equations of Rodriguez* are generalized and the result is used to obtain a simple expansion of a functional determinant of $\S 2$; in $\$ 4$, Theorem 1 of the previous article is generalized to the case of an $n$-tuple integral; in $\$ 5$, the results relative to the first variation in the previous article are generalized; in $\S 6$, two expressions for $I^{\prime \prime}(0)$ are given; in $\$ \$ 7$ and 8 , the boundary value problem and the discussion of the minimal surface, respectively, of the previous article are generalized; and the object of $\S 9$ is as we stated above.

We wish to thank Professor L. P. Eisenhart for numerous suggestions that he has given relative to $\$ \S 1,2$ of this paper.

2 . The normal coordinate system. $\dagger$ Let $L_{0}$ be a simply closed $(n-1)$ dimensional manifold with equations

$$
x_{i}=\xi_{i}(u)
$$$$
(i=1, \cdots, n) \text {, }
$$

where $u$ is $(n-1)$-partite and the $\xi$ 's are defined for all real values of the $u$ 's, are of class $C^{\prime \prime}$, have

$$
\sum_{i=1}^{n} \xi_{i u_{j}}^{2} \neq 0
$$$$
(j=1, \cdots, n-1)
$$

where the subscript $u_{j}$ indicates partial differentiation of the $\xi_{i}$ with respect to $u_{j}$ (similar subscript notation is used throughout the sequel) and each $\xi_{i}$ has in $u_{j}$ a period, say $t_{j}$, which is passed through once (exactly) when a point $x$ passes once-around the $u_{j}$-curve on $L_{0}$. We agree further, as stated above, to take the lines of curvature on $L_{0}$ as our parameter lines, so that the $u$-curves on $L_{0}$ are mutually orthogonal. $\ddagger$

We now introduce near $L_{0}$ a $u v$-coordinate system determined by the equations

$$
\text { (2.1) } \quad x_{i}=\xi_{i}(u)+v A_{i}
$$$$
(i=1, \cdots, n), 0 \leqq u_{i} \leqq t_{i}, v_{1} \leqq v \leqq v_{2},
$$

* Cf. Eisenhart's Differential Geometry, p. 122. to in $\$ 1$.

$\dagger$ Some of the ideas of this section are also expressed in $\$ 6$ of Powell's thesis, which was referred

‡ Cf., for example, Bates, loc. cit., p. 25, Theorem 1. 
where

$$
A_{i}=(-1)^{i-1} \frac{\partial\left(\xi_{1}, \cdots, \xi_{i-1}, \xi_{i+1}, \cdots, \xi_{n}\right)}{\partial\left(u_{1}, \cdots, u_{n-1}\right)} ;
$$

$\sum_{i=1}^{\infty} A_{i}^{2}=1$, a condition that can be realized by a suitable choice of parameters; $v$ is one-partite; and $v_{1}<0, v_{2}>0$ are sufficiently small numerically that there exist unique functions

$$
u_{j}=U,\left(x_{1}, \cdots, x_{n}\right), \quad v=V\left(x_{1}, \cdots, x_{n}\right)
$$

of class $C^{\prime}$ satisfying equations (2.1). This is possible* since for $x$ on $L_{0}$, $(u, v)=(u, 0)$ and the functional determinant

$$
\left.\Delta(v)\right|^{v=0}=\left|\begin{array}{ccc}
\xi_{1 u_{1}}+v A_{1 u_{1}}, & \cdots, & \xi_{n u_{1}}+v A_{n u_{1}} \\
\xi_{1 u_{n-1}}+v A_{1 u_{n-1}}, & \cdots & \xi_{n u_{n-1}}+v A_{n u_{n-1}} \\
A_{1} & , \cdots, & A_{n}
\end{array}\right|^{v=0}
$$

$$
=\left|\begin{array}{c}
\xi_{1 u_{1}}, \cdots, \xi_{n u_{1}} \\
\cdot \\
\xi_{1 u_{n-1}}, \cdots, \\
A_{1}, \cdots, \xi_{n u_{n-1}}
\end{array}\right|=(-1)^{n-1} \sum_{i=1}^{n} A_{i}^{2}=(-1)^{n-1} \neq 0 .
$$

We call our coordinate system normal because the $(n-1)$ lines of curvature $u_{j}$ are mutually orthogonal and, at every point $P$ of $L_{0}, v$ measures along the unique normal in the $X$ space to $L_{0}$ through $P$ for all values of $n \geqq 2$.

After obtaining, in $\$ 3$, a simple expansion of the determinant $\Delta(v)$, we shall employ, in $\$ 4$, the coordinate system of this section in differentiating an $n$-tuple integral with respect to a parameter.

3. Use of generalized equations of Rodriguez $\dagger$ in expanding $\Delta(v)$. The generalization of the equations of Rodriguez may be obtained from the first set of equations that Bates displays on page 24 of his article referred to above. In these equations we let $n$ be the $(n-1)$, number of $u$ 's, of the present paper, and we take his $x_{j}, \zeta_{j}^{i}$ as our $u_{j}, A_{i u_{j}}$, respectively. Since we are taking the lines $u_{j}$ to be lines of curvature (mutually orthogonal), we thus obtain the following generalized equations of Rodriguez:

$$
\rho_{j} A_{i u_{j}}=\xi_{i u_{j}}
$$

$$
(i=1, \cdots, n),
$$

where $\rho_{j}$ is, except possibly for sign, the radius of curvature of the $u_{j}$-line of

* Cf. G. A. Bliss, Princeton Colloquium Lectures, p. 20.

† Cf. Eisenhart's Differential Geometry, p. 122. 
curvature. As a consequence of (3.1), the determinant $\Delta(v)$ in (2.3) may be written

or

$$
\begin{aligned}
& \left|\begin{array}{ccc}
\xi_{1 u_{1}}\left(1+v / \rho_{1}\right) & , \cdots, \xi_{n u_{1}}\left(1+v / \rho_{1}\right) \\
\cdot & \cdot & \cdot \\
\xi_{1 u_{n-1}}\left(1+v / \rho_{n-1}\right) & \cdots, & \cdot \\
A_{1} & , \cdots, & \xi_{n u_{n-1}}\left(1+v / \rho_{n-1}\right) \\
\quad & & A_{n}
\end{array}\right| \\
& = \pm\left(1+v / \rho_{1}\right) \cdots\left(1+v / \rho_{n-1}\right) \sum_{i=1}^{n} A_{i}{ }^{2} ;
\end{aligned}
$$

$$
\Delta(v)= \pm\left(1+\pi_{1} v+\cdots+\pi_{n-1} v^{n-1}\right),{ }^{*}
$$

where $\pi_{t}$ is the elementary symmetric function of $t$ th order of the $\rho_{j}^{-1}$ $(j=1, \cdots, n-1)$.

4. The derivatives of an $n$-tuple integral with respect to a parameter. Consider a family of $(n-1)$-dimensional manifolds, one of which, $L_{a}$ (cf. the equation of $L_{0}$ in $\$ 2$ ), is given by the equations

$$
x_{i}=\xi_{i}+v(u, a) A_{i} \quad(i=1, \cdots, n),
$$

where $v(u, a)$ is defined and of class $C^{\prime \prime}$ for all $(u, a)$ having each $u_{j}$ as it was defined in $\$ 2$ and $a$ sufficiently near zero $\left(v_{1} \leqq v(u, a) \leqq v_{2}\right)$, where $v(u, a)$ has for each $u_{j}$ a period $T_{j}(a)$ that reduces to $T_{j}(0)=t_{j}$ for $a=0$; and $v(u, 0) \equiv 0$. All of the manifolds $L_{a}$ are closed on account of this periodicity and each $L_{a}$ is also simply closed for all values of $a$ sufficiently near zero since $L_{0}$ is simply closed. We let $S_{a}$ denote the hyperarea bounded by $L_{a}$ with the special understanding that when $a$ satisfies the equation $v(u, a)=v_{1}$, we are to designate $S_{a}$ and $L_{a}$ as $S_{1}$ and $L_{1}$, respectively.

Let $g(x, a)$ be a function of $a$ and the $x_{i}(i=1, \cdots, n)$ which is of class $C^{\prime \prime}$ for all sets $(x, a)$ having $x$ in a sufficiently small neighborhood of the hyperarea $S_{0}$ bounded by $L_{0}$ and having $a$ such that $v_{1} \leqq v(u, a) \leqq v_{2}$. Define $J(a)$ by the formula

$$
J(a)=\int_{s_{a}}^{n} g(x, a) d x .
$$

We desire the derivatives $J^{\prime}(0)$ and $J^{\prime \prime}(0)$. To obtain them, we first express the integral (4.2) as a sum of two integrals:

* The relative simplicity of this expansion may be observed by comparing it with the one that results if $\Delta(v)$, in (2.3), is expanded by minors in the notation that Bates used in a similar expansion (cf. Bates, loc. cit., equation (34)). 


$$
J(a)=\int_{S_{1}}^{n} g(x, a) d x+\int_{\Delta S}^{n} g(x, a) d x
$$

where $S_{1}$ is as it was defined in $\S 2$ and $\Delta S$ is the hyperarea in the $X$ space that is bounded by the $(n-1)$-dimensional manifolds $L_{1}$ and $L_{a}$. The derivative of the first integral in (4.3) has the value

$$
\int_{S_{1}}^{n} g_{a}(x, a) d x
$$

To differentiate the last integral in $\left(4 .{ }^{2}\right)$, we first transform it to the $u v$ coordinate system by means of (2.1). Letting $\Delta(v)^{+}$stand for the value of $\Delta(v)$ when the + sign is used before the parenthesis in (3.2), we find

$$
\int_{\Delta S}^{n} g(x, a) d x=\int_{L_{0}}^{n-1}\left[\int_{v_{1}}^{v(u, a)} g(\xi+v A, a) \Delta(v)+d v\right] d u,
$$

where $\xi+v A$ stands for the $n$ expressions $\xi_{1}+v A_{1}, \cdots, \xi_{n}+v A_{n}$. Since $a$ occurs only in the upper limit of the inner integral of (4.5) and explicitly in $g$, we find the derivative in question to be

$$
\int_{L_{0}}^{n-1} g v_{a} \Delta(v)+d u+\int_{L_{0}}^{n-1}\left[\int_{v_{1}}^{v(u, a)} g_{a} \Delta(v)+d v\right] d u,
$$

where in the first integral $v=v(u, a)$. Adding this result to the expression (4.4), we obtain

$$
\begin{aligned}
J^{\prime}(a)=\int_{S_{1}}^{n} g_{a} d x & +\int_{L_{0}}^{n-1}\left[\int_{v_{1}}^{v(u, a)} g_{a} \Delta(v)+d v\right] d u \\
& +\int_{L_{0}}^{n-1} v_{a} g(\xi+v A, a) \Delta(v)+d u .
\end{aligned}
$$

Hence, after transforming the second integral to $x$-coordinates, we find

$$
J^{\prime}(a)=\int_{S_{a}}^{n} g_{a} d x+\int_{L_{0}}^{n-1} v_{a} g(\xi+v A, a) \Delta(v)+d u .
$$

From the above procedure (perhaps with reference to the previous article), we now find without difficulty that

(4.7) $J^{\prime \prime}(a)=\int_{S_{a}}^{n} g_{a a} d x+\int_{L_{0}}^{n-1}\left[\left(g v_{a a}+2 g_{a} v_{a}+g_{v} v_{a}^{2}\right) \Delta(v)++g v_{a} \Delta_{a}(v)+\right] d u$.

Putting $a=0$ in (4.6) and (4.7) and recalling that $v(u, 0) \equiv 0$, we find the desired results, which we express as follows. 
TheORem 4.1. The derivatives $J^{\prime}(0)$ and $J^{\prime \prime}(0)$ of the n-tuple integral $J(a)$ defined by (4.2), taken over the n-dimensional region $S_{a}$ bounded by the manifold $L_{a}$, defined by equations (4.1), have the values

$$
\begin{aligned}
& J^{\prime}(0)=\int_{S_{0}}^{n} g_{a} d x+\int_{L_{0}}^{n-1} g v_{a} d u, \\
& J^{\prime \prime}(0)=\int_{S_{0}}^{n} g_{a a} d x+\int_{L_{0}}^{n-1}\left(g v_{a a}+\pi_{1} g v_{a}^{2}+2 g_{a} v_{a}+g_{v} v_{a}^{2}\right) d u .^{*}
\end{aligned}
$$

The derivatives (4.8) have been computed for the family of variations (4.1) of $L_{0}$. However we can obtain from (4.8) analogous formulas for a more general family of variations of $L_{0}$ of the form

$$
x_{i}=X_{i}(\tau, a)
$$$$
(i=1, \cdots, n) \text {, }
$$

where $\tau$ is $(n-1)$-partite and $\tau_{j}=\tau_{j}\left(u_{1}, \cdots, u_{n-1}, a\right)(j=1, \cdots, n-1)$, with $\tau_{j}\left(u_{1}, \cdots, u_{n-1}, 0\right)=u_{j}$. We suppose that (4.9) represents a one-parameter family of simply closed $(n-1)$-dimensional manifolds containing $L_{0}$ for $a=0$. The functions $X_{i}$ are supposed to be of class $C^{\prime \prime}$ for all values of $(\tau, a)$ having each $\tau_{j}$ real and $a$ sufficiently near zero. They have a period $\Gamma_{j}(a)$ for every $a$ that we consider, with $\Gamma_{j}(0)=t_{j}$. Such a family is representable in the form (4.1) if we can solve the equations

$$
X_{i}(\tau, a)-\xi_{i}(u)-v A_{i}(u)=0
$$

for $v$ and the $\tau_{j}$ as functions of $a$ and the $u_{j}$. According to the implicit function theorem used in $\$ 2$, this can be done since the equations (4.10) have the particular solution $(v, \tau, u, a)=(0, u, u, 0)$ for $0 \leqq u_{j} \leqq t_{j}$, on which the functional determinant

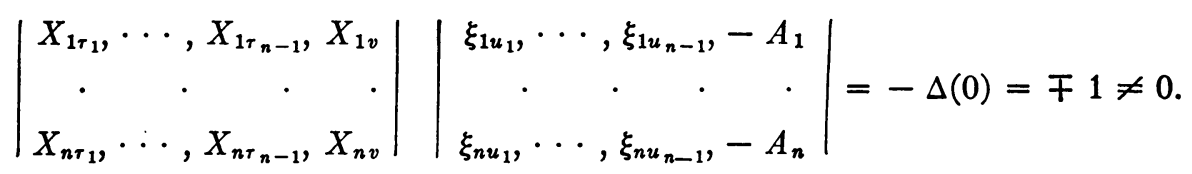

Hence we can obtain $v_{a}$ and $v_{a a}$ for the general family (4.9). Differentiating (4.10) once, twice, and agreeing that a term in which $j$ appears as a repeated index (even though it be a subscript of a subscript) is to be summed for all integral values of $j$ from 1 to $(n-1)$, and setting $a=0$, we obtain (4.11), (4.12), respectively,

$$
\begin{gathered}
X_{i \tau_{j}} \tau_{j a}-A_{i} v_{a}+X_{i a}=0 \\
X_{i \tau_{j} \tau_{j a a}}-A_{i} v_{a a}+X_{i a a}+2 X_{i a r_{j}} \tau_{j a}+X_{i \tau_{j} \tau_{k}} \tau_{j a} \tau_{k a}=0 .
\end{gathered}
$$

* In the second of equations (9) of the previous article there is a misprint. The last term of the integrand of the line integral of $J^{\prime \prime}(0)$ there should be $g_{v} v_{a}^{2}$. 
The determinant of the $n$ equations (4.11) in the $\tau_{j a}$ and $v_{a}$, like that of the $n$ equations (4.12) in the $\tau_{j a a}$ and $v_{a a}$, is $-\Delta(0)=\mp 1$. After a sense is assigned to $L_{0}$, so as to give $\Delta(0)$ a definite value (cf. (2.2)), say +1 , equations (4.11) define $v_{a}$ as a polynomial in the $X_{i a}$ and the $X_{i r_{j}}\left(=\xi_{i u_{j}}\right.$ on $L_{0}$ ), while (4.12) define $v_{a a}$ similarly in the $X_{i a a}, X_{i a \tau_{j}}, X_{i r_{j} \tau_{k}}, X_{i \tau_{j}}$, and $\tau_{j a}$. But the $\tau_{j a}$ in $v_{a a}$ can be eliminated by means of (4.11); the $\tau_{j a}$ are then polynomials in the $X_{i a}, X_{i \tau_{j}}$ since the $A_{i}$ are polynomials in the $X_{i \tau_{j}}$ (cf. the functional determinant last displayed above). Hence we have the following corollary of Theorem 4.1.

CoRollary. The derivatives $J^{\prime}(0)$ and $J^{\prime \prime}(0)$ in equations (4.8) can be generalized to the case where (4.1) is replaced by (4.9). When this is done, $v_{a}$ is a polynomial in the $X_{i a}$ and the $X_{i \tau_{j}}$, while $v_{a a}$ is a polynomial in the $X_{i a}, X_{i \tau_{j}}$, $X_{i a a}, X_{i a \tau_{j}}, X_{i \tau_{j} \tau_{k} \boldsymbol{k}}$.

5. The first variation. In the sequel, any term that contains a repeated index other than $a, v, z$, however it may appear, is to be summed for all integral values of the index from 1 to $n$. Thus we write $f_{p_{i}}\left(\phi_{x_{i}}+p_{i} \phi_{z}\right)$ for the sum of the $n$ terms that one obtains from this expression by taking $i=1, \cdots, n$. Further, when we use Kronecker $\delta$ 's with $\delta_{i}{ }^{k}=1$ or 0 according as $k=i$ or $k \neq i$, respectively, we extend customary convention by admitting subscripts of subscripts as summation indices; thus we would write $\zeta_{x_{k}} \delta_{i}{ }^{k}=\zeta_{x_{i}}$.

Proceeding now as we did in $\$ 3$ of the previous article, we find without difficulty the following equations, of which we number only those that are to be referred to later:

$$
\begin{gathered}
I^{\prime}(0)=\int_{S_{0}}^{n} f_{a} d x+\int_{L_{0}}^{n-1} f v_{a} d u, f_{a}=f_{z} \zeta+f_{p_{i}} \zeta_{x_{i}} ; \\
\phi(\xi+v A, z(\xi+v A)+a \zeta(\xi+v A))=0,
\end{gathered}
$$

$\xi+v(u, a) A$ being as in (4.5), so that $\phi$ contains the variables $u, v, a$;

$$
\begin{aligned}
v_{a} & =-\phi_{a} / \phi_{v} \\
\phi_{a} & =\phi_{z} \zeta, \quad \phi_{v}=\left(\phi_{x_{i}}+p_{i} \phi_{z}\right) A_{i} ; \\
v_{a} & =-\phi_{z} \zeta /\left(\phi_{x_{i}}+p_{i} \phi_{z}\right) A_{i} ; \\
I^{\prime}(0) & =\int_{S_{0}}^{n}\left(f_{z} \zeta+f_{p_{i}} \zeta x_{i}\right) d x+\int_{L_{0}}^{n-1} \frac{-\zeta f \phi_{z} d u}{\left(\phi_{x_{i}}+p_{i} \phi_{z}\right) A_{i}} .
\end{aligned}
$$

Hence we have the following theorem:

THEOREM 5.1. The first derivative $I^{\prime}(0)$ of the n-tuple integral (1.1), taken over the portion of the hypersurface $z=z(x)+a \zeta(x)$ bounded by its intersection with the hypersurface $\phi(x, z)=0$, has the value given by (5.4). 
From the point of view of the calculus of variations it is desirable to perform an integration by parts on the terms $f_{p_{i}} \zeta_{x_{i}}$ of (5.4). Since

$$
f_{p_{i}} \zeta_{x_{i}}=\frac{\partial}{\partial x_{i}}\left(f_{p_{i}} \zeta\right)-\zeta \frac{\partial}{\partial x_{i}} f_{p_{i}},
$$

we can replace the $n$-tuple integral in (5.4) by

$$
\begin{aligned}
\int_{S_{0}}^{n}\left[\zeta \left(f_{z}-\right.\right. & \left.\left.\frac{\partial}{\partial x_{i}} f_{p_{i}}\right)+\frac{\partial}{\partial x_{i}} f_{p_{i}} \zeta\right] d x \\
& =\int_{S_{0}}^{n} \zeta\left(f_{z}-\frac{\partial}{\partial x_{i}} f_{p_{i}}\right) d x+\int_{L_{0}}^{n-1} \zeta f_{p_{i}} A_{i} d u .^{*}
\end{aligned}
$$

Using this result in (5.4), we obtain

$$
I^{\prime}(0)=\int_{S_{0}}^{n} \zeta\left(f_{z}-\frac{\partial}{\partial x_{i}} f_{p_{i}}\right) d x+\int_{L_{0}}^{n-1} \zeta\left(\frac{\phi_{v} A_{i} f_{p_{i}}-f \phi_{z}}{\phi_{v}}\right) d u .
$$

Since, along $L_{0}^{\prime}, \phi\left(\xi_{1}(u), \cdots, \xi_{n}(u), z\left(\xi_{1}, \cdots, \xi_{n}\right)\right) \equiv 0$ in the $u_{j}$, we can replace (5.5) by an equivalent equation analogous to equation (32) of the previous article. Differentiating this identity, $\phi \equiv 0$, with respect to each $u_{j}$, we obtain the $(n-1)$ equations

$$
\left(\phi_{x_{i}}+p_{i} \phi_{z}\right) \xi_{i u_{j}}=0 \quad(j=1, \cdots, n-1) .
$$

Hence with (5.6) and the last equation in (5.2), we have $n$ equations which determine the $\phi_{x_{i}}+p_{i} \phi_{z}$ :

$$
\phi_{x_{i}}+p_{i} \phi_{\varepsilon}=A_{i} \phi_{v}(\mathrm{cf} .(2.2)),
$$

where

$$
\phi_{v}^{2}=\sum_{i=1}^{n}\left(\phi_{x_{i}}+p_{i} \phi_{z}\right)^{2} \neq 0 .
$$

That $\phi_{v}{ }^{2} \neq 0$ may be proved as follows. Suppose $\phi_{v}^{2}=0$, so that

$$
\phi_{x_{i}}+p_{i} \phi_{z}=0 \quad(i=1, \cdots, n) .
$$

Then the hypersurfaces $z=z(x)$ and $\phi(x, z)=0$ are tangent to each other, which is impossible when $f \neq 0$ along $L_{0}^{\prime}$, as we shall see just below Corollary 5.2. In view of (5.7), (5.5) is equivalent to

$$
I^{\prime}(0)=\int_{S_{0}}^{n} \zeta\left(f_{z}-\frac{\partial}{\partial x_{i}} f_{p_{i}}\right) d x+\int_{L_{0}}^{n-1} \zeta \frac{\left[f_{p_{i}}\left(\phi_{x_{i}}+p_{i} \phi_{z}\right)-f \phi_{z}\right] d u}{\left(\phi_{x_{i}}+p_{i} \phi_{z}\right) A_{i}}
$$

* In obtaining this term we have used the extended Green's theorem referred to above with the $A_{i}$ as direction cosines of the outer normal to $L_{0}$. 
The Euler necessary condition for a minimum value of $I$ in the case of fixed limits (where the hypersurface $\phi(x, z)=0$ is replaced by a bounded, closed, connected $(n-1)$-dimensional manifold, such as $\left.L_{0}\right)$ is

$$
f_{z}-\frac{\partial}{\partial x_{i}} f_{p_{i}}=0^{*}
$$

at every point of $S_{0}$. This is surely a necessary condition for the case of variable limits. Since $I^{\prime}(0)=0$ is a necessary condition for a minimum value of $I$, it now follows that if $z=z(x)$ minimizes $I$ the second integral in (5.9) vanishes, and indeed that the numerator, $N$, of the integrand of this integral is zero at every point of $L_{0}, 0 \leqq u_{j} \leqq t_{j}$, as we presently prove. Suppose $N$ does not so vanish. Either $N$ has one sign on the entire manifold $L_{0}$ or there is a nonzero $(n-1)$-dimensional subregion of $L_{0}$ on which $N$ has one sign. We may take $\zeta\left(u_{1}, \cdots, u_{n-1}\right)$ to be of the same sign as $N$ on one such subregion and zero elsewhere. Then since $\phi_{v} \neq 0, I^{\prime}(0) \neq 0$ (contradiction). Hence we have the transversality condition

$$
f_{p_{i}}\left(\phi_{x_{i}}+p_{i} \phi_{z}\right)-f \phi_{z}=0
$$

at every point of $L_{0}$.

We now have the following corollaries of Theorem 5.1.

Corollary 5.1. The first derivative of the n-tuple integral $I(a)$, of (1.2), taken over the portion of the hypersurface $z=z(x)+a \zeta(x)$ bounded by its intersection with the fixed hypersurface $\phi(x, z)=0$, has the value given by (5.9).

COROLLARY 5.2. In case $z=z(x)$ is a minimizing hypersurface for the $n$-tuple integral (1.1), the Euler equation (5.10) must hold at every point of the portion of the hypersurface $z=z(x)$ inside $L_{0}^{\prime}$, and the transversality condition (5.11) must hold at every point of the boundary, $L_{0}^{\prime}$, which is the manifold of intersection of the hypersurfaces $z=z(x)$ and $\phi(x, z)=0$.

Since we have assumed in $\$ 1$ that $f \neq 0$ along $L_{0}^{\prime}$, it follows from (5.11) that the hypersurface $z=z(x)$ is not tangent to the hypersurface $\phi(x, z)=0$ at any point of $L_{0}^{\prime}$. In the case of the minimal hypersurface for which $f=(1$ $\left.+p_{t} p_{t}\right)^{1 / 2},(5.11)$ reduces to $p_{i} \phi_{x_{i}}-\phi_{z}=0$, which shows that the hypersurfaces $z=z(x)$ and $\phi(x, z)=0$ meet at right angles.

6 . The second variation. To get $I^{\prime \prime}(0)$, we apply to (1.2) the result in the second of equations (4.8). Replacing $g$ in that equation by $f$, we obtain

$$
I^{\prime \prime}(0)=\int_{S_{0}}^{n} f_{a a} d x+\int_{L_{0}}^{n-1} M d u,
$$

\footnotetext{
* Cf.page 5 of Powell's thesis, loc. cit.
} 
where $M \equiv f v_{a a}+f \pi_{1} v_{a}^{2}+f_{v} v_{a}^{2}+2 f_{a} v_{a}$ and

$$
f_{a a}=f_{z z} \zeta^{2}+2 f_{z p_{i}} \zeta \zeta_{x_{i}}+f_{p_{i} p_{j}} \zeta_{x_{i}} \zeta_{x_{j}} .
$$

By differentiating the equation $\phi_{v} v_{a}+\phi_{z}=0$ (cf. (5.1)), with respect to $a$, we find, as in the previous article, that

$$
v_{a a}=-\frac{1}{\phi_{v}}\left(\phi_{v v} v_{a}^{2}+2 \phi_{a v} v_{a}+\phi_{a a}\right),
$$

where $\phi_{v}$ is defined in (5.2), and $\phi_{v v}, \phi_{a v}, \phi_{a a}$, at $a=0$, are obtained by differentiating $\phi$ as a function of the arguments

$$
\xi_{i}+v A_{i}(i=1, \cdots, n), \quad z(\xi+v A)+a \zeta(\xi+v A),
$$

$\xi+v A$ standing for the set of $n$ expressions $\xi_{i}+v A_{i}$. We find, for $a=0$,

$$
\begin{aligned}
& \phi_{v v}=\left(\phi_{x_{i} x_{j}}+2 \phi_{x_{i} z} p_{j}+\phi_{z z} p_{i} p_{j}+\phi_{z} r_{i j}\right) A_{i} A_{j}, \quad r_{i j} \equiv z_{x_{i} x_{j}} \\
& \phi_{a v}=\left(\phi_{x_{i z}} \zeta+\phi_{z z} \zeta p_{i}+\phi_{z} \zeta x_{i}\right) A_{i} ; \quad \phi_{a a}=\phi_{z z} \zeta^{2} .
\end{aligned}
$$

Using these derivatives together with (5.2) and (5.3), we find (cf. (6.3))

$$
\begin{aligned}
v_{a a}= & -\frac{1}{\phi_{v}^{3}}\left\{\phi_{z}^{2} \zeta^{2}\left[\phi_{x_{i} x_{j}}+2 \phi_{x_{i} z} p_{j}+\phi_{z z} p_{i} p_{j}+\phi_{z} r_{i j}\right]\right. \\
& -2 \phi_{z} \zeta\left[\zeta\left(\phi_{x_{i} z}+p_{i} \phi_{z z}\right)\left(\phi_{x_{j}}+p_{j} \phi_{z}\right)\right. \\
& \left.\left.+\phi_{z}\left(\phi_{x_{j}}+p_{i} \phi_{z}\right) \zeta x_{x_{i}}\right]+\phi_{z z} \zeta^{2}\left(\phi_{x_{i}}+p_{i} \phi_{z}\right)\left(\phi_{x_{j}}+p_{j} \phi_{z}\right)\right\} A_{i} A_{j} .
\end{aligned}
$$

Hence if we collect in (6.4) the terms involving the second derivatives of $\phi$, those involving the second derivatives of $z$, and those free of second derivatives, we find

$$
\begin{aligned}
v_{a a}= & -\frac{\zeta^{2}}{\phi_{v}{ }^{3}}\left(\phi_{z}^{2} \phi_{x_{i} x_{j}}-2 \phi_{z} \phi_{x_{j}} \phi_{x_{i} z}+\phi_{z z} \phi_{x_{i}} \phi_{x_{j}}\right) A_{i} A_{j}-\frac{\phi_{z}^{3} \zeta^{2}}{\phi_{v}{ }^{3}} \cdot r_{i j} A_{i} A_{i} \\
& +\frac{2 \zeta \zeta_{x_{i}}}{\phi_{v}{ }^{3}}\left(\phi_{z}{ }^{2} \phi_{x_{j}}+\phi_{z}{ }^{3} p_{j}\right) A_{i} A_{j} .
\end{aligned}
$$

Now using the notation

$$
\begin{gathered}
q_{j}=-\phi_{x_{j}} / \phi_{z}, \quad \Delta=\left[\left(p_{i}-q_{i}\right)\left(p_{i}-q_{i}\right)\right]^{1 / 2}, \\
s_{i j}=-\frac{\phi_{z}^{2} \phi_{x_{i} x_{j}}-2 \phi_{z} \phi_{x_{j}} \phi_{x_{i} z}+\phi_{z z} \phi_{x_{i}} \phi_{x_{j}}}{\phi_{z}{ }^{3}}
\end{gathered}
$$

so that $\left(\partial q_{i} / \partial x_{j}\right)=\left(s_{i j}+s_{j i}\right) / 2$, we find from (6.5) that

$$
v_{a a}=\frac{1}{\Delta^{3}}\left[\zeta^{2}\left(s_{i j}-r_{i j}\right)+2 \zeta \zeta_{x_{i}}\left(p_{j}-q_{j}\right)\right] A_{i} A_{j} .
$$


The introduction of the $q_{i}$ and the $s_{i j}$ (whose denominators involve $\phi_{z}{ }^{8}$ ) does not require that $\phi(x, z)=0$ be representable in the form $z=z_{1}(x)$ (cf. the $(n-1)$-tuple integral in (6.9), in which $\Delta \neq 0$ since $\left.0 \neq \phi_{v}=\phi_{z} \Delta\right)$.

The other three terms of $M$ are

where

$$
\begin{aligned}
f v_{a}^{2} \pi_{1} & =\frac{f \zeta^{2}}{\Delta^{2}}\left(\frac{1}{\rho_{1}}+\cdots+\frac{1}{\rho_{n-1}}\right), \\
f_{v} v_{a}^{2} & =\frac{\zeta^{2}}{\Delta^{3}}(f)_{x_{i}}\left(p_{i}-q_{i}\right), \\
2 f_{a} v_{a} & =-(2 \zeta / \Delta)\left(f_{z} \zeta+f_{p_{i}} \zeta x_{i}\right),
\end{aligned}
$$

$$
(f)_{x_{i}}=f_{x_{i}}+f_{z} p_{i}+f_{p_{j}} r_{i j} .
$$

Collecting the terms of $M$, as they are given by (6.6), (6.7), (6.8), and using the value (6.2) of $f_{a a}$, we obtain the following theorem.

THEOREM 6.1. The second derivative $I^{\prime \prime}(0)$ of the n-tuple integral $I(a)$ of equation (1.2), taken over the portion of the hypersurface $z=z(x)+a \zeta(x)$ bounded by its intersection with the hypersurface $\phi(x, z)=0$, has the value

$$
I^{\prime \prime}(0)=\int_{S_{0}}^{n} 2 \Omega d x+\int_{L_{0}}^{n-1}\left(\zeta / \Delta^{8}\right)\left(B \zeta+C_{i} \zeta x_{i}\right) d u,
$$

where

$$
\begin{aligned}
2 \Omega & \equiv f_{z z} \zeta^{2}+2 f_{z p_{i}} \zeta \zeta_{x_{i}}+f_{p_{i} p_{j}} \zeta x_{i} \zeta_{x_{j}}, \\
B & \equiv f\left(s_{i j}-r_{i j}\right) A_{i} A_{j}+f \Delta \pi_{1}+(f)_{x_{i}}\left(p_{i}-q_{i}\right)-2 f_{z} \Delta^{2}, \\
C_{i} & \equiv 2\left[f\left(p_{i}-q_{j}\right) A_{j} A_{i}-f_{p_{i}} \Delta^{2}\right] .
\end{aligned}
$$

7. A boundary value problem associated with the second variation. We generalize the boundary value problem of the previous article. By Euler's theorem on homogeneous functions, the $n$-tuple integral of (6.9) can be written in the form

$$
\int_{S_{0}}^{n}\left(\zeta \Omega_{\zeta}+\zeta x_{i} \Omega_{i}\right) d x ; \quad \Omega_{i} \equiv \Omega_{x_{i}}
$$

Then, after performing a customary integration by parts, we find

$$
\int_{S_{0}}^{n} 2 \Omega d x=\int_{S_{0}}^{n}\left[\zeta \psi(\zeta)+\frac{\partial}{\partial x_{i}} \zeta \Omega_{i}\right] d x, \psi(\zeta) \equiv \Omega_{\zeta}-\frac{\partial}{\partial x_{i}} \Omega_{i} .
$$

Applying the extended Green's theorem heretofore used, we now find 


$$
\int_{S_{0}}^{n} 2 \Omega d x=\int_{S_{0}}^{n} \zeta \psi(\zeta) d x+\int_{L_{0}}^{n-1} \zeta A_{i} \Omega_{i} d u
$$

From (6.9) and (7.1), we now obtain

$$
I^{\prime \prime}(0)=\int_{s_{0}}^{n} \zeta \psi(\zeta) d x+\int_{L_{0}}^{n-1} \zeta\left(B \zeta+C_{i} \zeta_{x_{i}}+A_{i} \Omega_{i}\right) d u
$$

or since

$$
\Omega_{i}=\zeta f_{z p_{i}}+\zeta{ }_{x_{j}} f_{p_{i} p_{j}}
$$

we have

$$
I^{\prime \prime}(0)=\int_{S_{0}}^{n} \zeta \psi(\zeta) d x+\int_{L_{0}}^{n-1} \zeta\left(D \zeta+E_{i} \zeta{x_{i}}_{i}\right) d u
$$

where

$$
D \equiv B+A_{i} f_{s p_{i}}, \quad E_{i} \equiv C_{i}+A_{j} f_{p_{j} p_{i}} \text { (cf. (6.10)). }
$$

From (7.2) we can now state a new necessary condition in order that the hypersurface $z=z(x)$ shall minimize the $n$-tuple integral (1.1).

THEOREM 7.1. In order that the hypersurface $z=z(x)$ shall minimize the n-tuple integral (1.1), it is necessary that for negative values of $\lambda$ the boundary value problem

$$
\begin{aligned}
\psi(\zeta)-\lambda \zeta & =0 \text { in the region } S_{0}, \\
D \zeta+E_{i} \zeta_{x_{i}} & =0 \text { on the boundary } L_{0} \text { of } S_{0}
\end{aligned}
$$

have no solution except $\zeta \equiv 0, D$ and the $E_{i}$ being defined through (6.10) and (7.3).

8. The minimal hypersurface. Here we define $f=\left(1+p_{t} p_{t}\right)^{1 / 2}$. We shall compute the $I^{\prime \prime}(0)$ of $(6.9)$ for the present case. Since $f_{z}=0$, the only derivatives needed here are

$$
\begin{aligned}
& f_{p_{i}}=p_{i} / f, f_{p_{i} p_{j}}=\left(f^{2} \delta_{i}^{j}-p_{i} p_{j}\right) / f^{3},(f)_{x_{i}}=p_{j} r_{i j} / f(\mathrm{cf} .(6.8)), \\
& f_{a a}=2 \Omega=\zeta \Omega_{\xi}+f_{p_{i} p_{j}} \zeta_{x_{i}} \zeta_{x_{j}}=\zeta_{x_{i}} \zeta_{x_{j}}\left(f^{2} \delta_{i}^{j}-p_{i} p_{j}\right) / f^{3}
\end{aligned}
$$

One now finds that the $B, C_{i}$ of $(6.10)$ reduce to $B^{\prime}, C_{i}^{\prime}$, respectively, where

$$
\begin{aligned}
B^{\prime} & =f\left(s_{i j}-r_{i j}\right) A_{i} A_{j}+f\left(\frac{1}{\rho_{1}}+\cdots+\frac{1}{\rho_{n-1}}\right) \Delta+p_{j} r_{i j}\left(p_{i}-q_{i}\right) / f \\
C_{i}^{\prime} & =(2 / f)\left[f^{2}\left(p_{j}-q_{j}\right) A_{j} A_{i}-p_{i} \Delta^{2}\right] .
\end{aligned}
$$

Hence we have the following corollary of Theorem 6.1. 
COROLLARY 8.1. In the case of the minimal hypersurface, the $I^{\prime \prime}(0)$ of Theorem 6.1 reduces to

$$
I^{\prime \prime}(0)=\int_{S_{0}}^{n}\left(\zeta_{x_{i}} \zeta_{x_{j}} / f^{3}\right)\left(f^{2} \delta_{i}^{j}-p_{i} p_{j}\right) d x+\int_{L_{0}}^{n-1} \zeta\left(B^{\prime} \zeta+C_{i}^{\prime} \zeta_{x_{i}}\right) d u,
$$

where $B^{\prime}$ and the $C_{i}^{\prime}$ are as defined in (8.1).

To make a similar specialization of Theorem 7.1, we observe that in the present case

$$
\begin{aligned}
\psi(\zeta) & =-\frac{\partial}{\partial x_{i}} \Omega_{i}=-\frac{\partial}{\partial x_{i}} \zeta_{x_{j}}\left(f^{2} \delta_{i}^{j}-p_{i} p_{j}\right) / f^{3}, \\
D & =B^{\prime} \text {, of }(8.1), \\
E_{i} & =C_{i}^{\prime}+A_{j}\left(f^{2} \delta_{i}^{j}-p_{i} p_{j}\right) / f^{3}(\mathrm{cf} .(7.3) \text { and (8.1)). }
\end{aligned}
$$

Consequently we have the following corollary to Theorem 7.1.

COROLLARY 8.2. In order that the hypersurface $z=z(x)$ shall minimize the $n$-tuple integral (1.1) in the case where $f=\left(1+p_{t} p_{t}\right)^{1 / 2}$, it is necessary that for negative values of $\lambda$ the following boundary value problem have no solution except $\zeta \equiv 0$ :

where

$$
\begin{aligned}
& \frac{\partial}{\partial x_{i}} \Omega_{i}+\lambda \zeta=0 \text { on } S_{0} \\
& B^{\prime} \zeta+E_{i} \zeta_{x_{i}}=0 \text { on } L_{0}
\end{aligned}
$$

$$
-\frac{\partial}{\partial x_{i}} \Omega_{i}=\psi(\zeta)
$$

by (8.2), $B^{\prime}$ is defined by (8.1), and the $E_{i}$ are given in (8.2).

9. Further applications of Theorem 4.1. Since $g$ in $\$ 4$ is merely required to be a function of class $C^{\prime \prime}$ in the $x$ 's and the parameter $a$, there may be numerous applications of Theorem 4.1, even to more complicated variation problems than the problem associated with $\$ \$ 1-8$ above. We have made one such application. We have used the first equation of (4.8) to compute the first variation of the integral

$$
K=\int_{S_{0}}^{n} f(x, z, p, r) d x
$$

where $S_{0}, n, x, z, p$ have the meaning relative to $K$ which they had for $I$ in $\$ \S 1-8 ; r$ is the set of all of the derivatives

$$
\frac{\partial p_{i}}{\partial x_{j}}=r_{i j} \quad(i, j=1, \cdots, n) ;
$$


and $f$ is supposed to have suitable continuity in a region $W$ of the space $X Z P R$ in which a minimum value of $K$ is desired. A fixed hypersurface $\phi(x, z)=0$, with suitable continuity, is employed as in $\$ \$ 1-8$.

We state without proof that the analogs of (5.4) and (5.9) here are (9.1) and (9.2) below, respectively:

$$
\begin{aligned}
(9.1) K^{\prime}(0)= & \int_{S_{0}}^{n}\left(f_{z} \zeta+f_{p_{i}} \zeta x_{i}+f_{r_{i j}} \zeta_{x_{i} x_{j}}\right) d x-\int_{L_{0}}^{n-1} \frac{f \zeta \phi_{z} d u}{\left(\phi_{x_{i}}+p_{i} \phi_{z}\right) A_{i}} \\
\text { (9.2) } K^{\prime}(0)= & \int_{S_{0}}^{n} \zeta\left(f_{z}-\frac{\partial}{\partial x_{i}} f_{p_{i}}+\frac{\partial^{2} f_{r_{i j}}}{\partial x_{i} \partial x_{j}}\right) d x \\
& +\int_{L_{0}}^{n-1}\left[\left(f_{p_{i}} \zeta+f_{r_{i j}} \zeta x_{x_{j}}\right)\left(\phi_{x_{i}}+p_{i} \phi_{z}\right)-\zeta\left(\phi_{x_{j}}+p_{j} \phi_{z}\right) \frac{\partial}{\partial x_{i}} f_{r_{i j}}\right. \\
& \left.-\phi_{z} \zeta f\right] \cdot \frac{d u}{\left(\phi_{x_{i}}+p_{i} \phi_{z}\right) A_{i}} .
\end{aligned}
$$

By methods that were used in preceding sections, one could compute $K^{\prime \prime}(0)$.

NORTHWESTERN UNIVERSITY,

Evanston, Ill. 